se regeln können. Schließlich fand der Aufstand in Ungarn zur gleichen Zeit statt wie die Suezfrage.

Chruschtschow: Und das ist kein Zufall.

Bevan: Wenn die UdSSR davon überzeugt gewesen wäre, dass die Vereinigten Staaten vom bevorstehenden Überfall auf Ägypten Kenntnis hatten, hätte dies Auswirkungen auf die ungarische Frage gehabt. Doch die Engländer und die Franzosen haben in dieser Frage die Vereinigten Staaten hinters Licht geführt.

Chruschtschow: Das ist nicht seriös. Alle haben von der Aggressionsvorbereitung gewusst.

Bevan: Wir wissen, dass die USA von der Vorbereitung gewusst, aber nicht geglaubt haben, dass England und Frankreich Ägypten angreifen würden.

Chruschtschow: Darin kann ich Ihnen nicht zustimmen.

Zum Abschluss dankte Bevan N. S. Chruschtschow für das Gespräch und sagte, dass es ihm bei der weiteren Arbeit helfen wird.

Aufgezeichnet von Ju. Koroljow und W. Michajlow

Korrigiertes Manuskript

Übersetzt aus dem russischen Originaltext

RGANI, fond 57, opis' 1 , delo 569, Bl. 1-17

\title{
22. Gespräch Chruschtschows mit dem britischen Physiker J[ohn] Bernal am 11. November 1957
}

Zu Beginn des Gesprächs dankte J[ohn] Bernal417 Gen. Chruschtschow, dass er trotz seiner knapp bemessenen Zeit die Möglichkeit gefunden hat, ihn zu empfangen. Weiter sagte er, er würde gern Fragen der Politik von Frieden und Krieg erörtern, wie es darum jetzt steht, und auch über die Probleme sprechen, vor denen die Bewegung der Anhänger des Friedens 418 in der Welt und in England steht. Bernal bemerkte, der erfolgreiche Start der sowjetischen Sputniks ${ }^{419}$ und die Demonstration der Macht der Sowjetunion hätten in letzter Zeit zur Veränderung der Lage in der Welt geführt. Das wiederum führe zum Gegenangriff sei-

417 John Desmond Bernal, ein katholisch sozialisierter Sohn eines irischen Bauern und einer amerikanischen Protestantin, war ein international berühmter Physiker, der zahlreiche Auszeichnungen und Preise erhielt. Unter anderem war er Mitglied der Akademie der Wissenschaften der UdSSR und vieler anderer kommunistischer Staaten. An der Lomonosov-Universität in Moskau wurde er zum Professor ehrenhalber und an der Humboldt-Universität in Ost-Berlin zum Doktor ehrenhalber ernannt. 1953 verlieh ihm die Sowjetunion den Lenin-Friedenspreis.

418 Nach sowjetischer Auffassung gehörten dazu nur diejenigen Teile der Friedensbewegung, die jeweils die Bestrebungen des sowjetischen „Friedenslagers“ unterstützten.

419 Am 4. Oktober 1957 hatte die UdSSR vor den USA einen künstlichen Satelliten (sputnik) ins Weltall geschickt, dem ein weiterer folgte, und damit den - irrigen - Eindruck der Überlegenheit in der Raketentechnik erweckt. 
tens der amerikanischen und englischen politischen Kräfte. Auf der bevorstehenden Pariser Tagung der Mitgliedsländer des Nordatlantikpakts habe man die Absicht, einen Vereinigten Generalstab zu schaffen, die Bewaffnung und die Ausbildung der Truppen zu vereinheitlichen - mit einem Wort, ein noch engeres Militärbündnis zu schaffen. Solche Pläne werden einen weiteren Verlust an militärischer Selbständigkeit Englands bedeuten.

Gegenwärtig haben sich auf dem Gebiet der Militärtechnik gewaltige Veränderungen vollzogen. Im Zusammenhang damit ist in England und in den USA eine neue Theorie aufgetaucht, die Theorie des „begrenzten Atomkriegs“. Ihre Gefährlichkeit besteht darin, dass die Verfechter dieser Theorie davon überzeugt sind, dass sie sich erfolgreich anwenden lässt mittels der Entfesselung von lokalen Kriegen. Doch das kann seinerseits zum umfassenden und ernstlichen Krieg führen, wie es beinahe in Syrien geschehen wäre. Ich möchte Ihnen nichts von der komplizierten politischen Situation in der Welt und der gefährlichen Theorie des „begrenzten Krieges“ erzählen. Sie wissen das besser als ich. Ich möchte die Gelegenheit dieses Treffens dazu benutzen, Ihnen einige Fragen zu stellen. Auf welche Weise sollten wir, die Anhänger des Friedens, uns nach Ihrer Ansicht gegen diese Theorie wenden? Dadurch, dass sich die Bewegung der Anhänger des Friedens gegen sie und gegen die Beschlüsse der bevorstehenden Pariser Beratung der Mitgliedsländer der NATO wendet, lässt sich viel machen. In England gibt es die Möglichkeit, eine beinahe offizielle Kampagne unter Beteiligung von Vertretern der Labour Party für den Verzicht auf die Pariser Verträge ${ }^{420}$ zu organisieren. Jetzt spricht sich Bevan ${ }^{421}$ offiziell für einen Versuchsstopp und ein Verbot der Atomwaffen aus. Doch kann man sich der Labour-Politiker nie sicher sein, und sogar Bevan bezieht eine unklare Position. Zudem gibt es in der Labour Party Elemente, die für ein Militärbündnis mit den USA eintreten. Doch die englischen Anhänger des Friedens sollten die Kampagne durchführen, um einige Labour-Politiker auf ihre Seite zu ziehen und noch vor Dezember, wenn die Pariser [NATO-]Konferenz beginnt, eine breite Opposition gegen ihre Beschlüsse zu organisieren. Natürlich werden der Erfolg unseres Kampfes und das Ausmaß unserer Unterstützung für die Außenpolitik der UdSSR in großem Umfang von der Position und den Stellungnahmen der Sowjetunion abhängen. Ich habe mich mit Ihrer Rede auf der Tagung des Obersten Sowjets der UdSSR 422 befasst und

420 Aufgrund der Pariser Verträge vom 23. Oktober 1954 war die Bundesrepublik Deutschland am 5. Mai 1955 in die NATO aufgenommen worden.

421 Aneurin Bevan, führender Exponent des linken Flügels der Labour Party.

422 Chruščëv hatte in seiner Rede vor dem Obersten Sowjet zum 40. Jahrestag der Oktoberrevolution am 6. November 1957 die Leistungen der sowjetischen Forschung und Technik und die die Bedeutung der neuen sozialistischen Gesellschaft hervorgehoben sowie unter Hinweis auf den XX. Parteitag der KPdSU als großen Wendepunkt Kritik an Stalin geübt, doch davor gewarnt, die Kritik zu weit zu treiben und ,zu Ausfällen gegen die sozialistische Gesellschaftsordnung ... zu missbrauchen“. Die Partei vervollkommne sich unter strikter Anwendung der Leninschen Grundsätze und gestehe auch ihre Fehler ein. Ohne sie wäre das Volk nicht in der Lage gewesen, die Macht zu erringen und die sozialistische Gesellschaft aufzubauen. Chruščëv bezeichnete das Weltsystem der sozialistischen Staaten als Triumph des Marxismus-Leninismus und betonte die Unterschiede zum „kapitalistischen Weltsystem“, brachte jedoch die Ansicht zum Ausdruck, dass die - von Lenin begründete - friedliche Koexistenz zur „historischen Notwendigkeit“ geworden sei. Eine sachliche Verständigung auf dieser Grundlage ermögliche eine Lösung der großen internationalen Probleme. Die Vertreter der kapitalistischen und der sozialistischen Länder 
denke, dass sie nützlich ist und ein positives Echo in der Weltöffentlichkeit finden wird. Die darin dargelegte Haltung der Sowjetunion wird zur Stärkung der Bewegung der Anhänger des Friedens und zur Schwächung derjenigen beitragen, die für eine militärisch ausgerichtete Politik sind. Aktionen zugunsten des Friedens in den Ländern der Volksdemokratie werden es den Friedenskräften in der Welt ermöglichen, sich um die Länder des sozialistischen Lagers zu scharen.

Weiter möchte ich eine Frage ansprechen, zu der Sie keinen direkten Bezug haben. Das ist die Ungarn-Frage. Inzwischen ist der negative Einfluss der Ereignisse vom letzten Jahr 423 überwunden, doch in einigen Kreisen der Gesellschaft ist erneut Irritation aufgekommen im Zusammenhang mit Unterdrückungsmaßnahmen der ungarischen Regierung gegenüber einigen ungarischen Schriftstellern. Vom Standpunkt der Ungarn selbst sind diese Maßnahmen offenbar berechtigt, doch die Öffentlichkeit im Westen ist besorgt, und das Vorgehen der ungarischen Behörden kann in gewissem Umfang von den Feinden gegen die Sowjetunion ausgenutzt werden. Doch diese Frage ist nicht die Hauptsache, und ich möchte zur folgenden Frage übergehen.

Kürzlich ist in den Vereinigten Staaten ein offizielles oder offiziöses Buch erschienen, dessen Autor, Kissinger, einer der Führer der Luftstreitkräfte der USA ist und sich der Unterstützung von Dulles erfreut. ${ }^{424}$ Die Kernfrage dieses Buches ist eine Theorie des sogenannten „begrenzten Atomkriegs“. Der Autor des Buches versichert, die Atomwaffe sei lediglich eine Waffe zur Einschüchterung ${ }^{425}$ und werde nicht zum Einsatz kommen. Das Buch enthält weiter die Behauptung, die USA seien gezwungen gewesen, die Atomwaffe unter dem Einfluss des Drucks seitens der Sowjetunion zu entwickeln, die über die Bewegung der Anhänger des Friedens verfüge. Ich denke, dass wir trotz dieser Mutmaßungen den Kampf der Anhänger des Friedens gegen den Atomwaffengebrauch verstärken müssen. Die Bewegung der Anhänger des Friedens kann in dieser Frage eine positive Rolle spielen. Sie hat sich jetzt von den Schwierigkeiten erholt, in denen sie sich vor einem Jahr befunden hat.426 Wenn wir jetzt einen Kongress der Anhänger des Friedens erfolgreich durchführen könnten, würde dies die Bewegung erheblich stärken. Es ist nötig, dass die Sowjetunion unserer Bewegung mehr Unterstützung gibt. Ich will damit nicht sagen, dass die Bewegung der Anhänger des Friedens von der Sowjetunion aus geführt werden sollte, aber an der Bewegung sollten sich mehr

sollten darüber auf einer hochrangigen Konferenz zu einem Einvernehmen gelangen. Dem stehe freilich die aggressive Politik der NATO-Staaten entgegen. Die imperialistischen Monopole seien aus wirtschaftlichen Gründen gegen Abrüstung und Entspannung. Der ideologische Gegensatz sei unüberbrückbar, doch wollten die Kommunisten ihre Ziele niemals mittels Gewalt erreichen.

${ }^{423}$ Der Ungarn-Aufstand und die daraufhin erfolgte sowjetische Intervention im Herbst 1956.

${ }^{424}$ Gemeint ist offenbar Henry Kissinger, der freilich kein Kommando bei der U.S. Air Force inne hatte, sondern zu dieser Zeit Professor an der Harvard University und Mitglied verschiedener politischer Beratungsgremien war. Er hatte Kontakte zu führenden republikanischen Politikern geknüpft und 1956 ein aufsehenerregendes Werk „Nuclear Weapons and Foreign Policy“ publiziert.

425 Mit diesem Ausdruck wurde in der UdSSR die westliche Vorstellung der Abschreckung bezeichnet. Es ist daher zu vermuten, dass Bernal an dieser Stelle das Wort „deterrence“ verwendete, das vom sowjetischen Übersetzer und Protokollanten mit „Einschüchterung“ (zapugivanie) wiedergegeben wurde.

426 Im Herbst 1956 war hatte die sowjetische Militäraktion in Ungarn auch in pazifistischen Kreisen heftige Kritik hervorgerufen. 
sowjetische Vertreter beteiligen. Unsere Freunde Ehrenburg427 und Kornejčuk ${ }^{428}$ sind in der Bewegung sehr aktiv, doch das ist nicht genug, es müssen sich [noch] mehr sowjetische Vertreter an der Bewegung beteiligen. Sie wissen sicherlich, dass es im Weltfriedensrat ${ }^{429}$ eine Opposition gegen D’Astier de la Vigerie ${ }^{430}$ gibt. Die Bewegung der Anhänger des Friedens kann aber auf ihn nicht verzichten, denn nur er hat die Fähigkeit, die unterschiedlichen Kräfte aneinander anzunähern, und ohne ihn hätte sich die Bewegung unweigerlich gespalten. Vor uns steht jetzt die Frage, wie die Tätigkeit der Anhänger des Friedens neu zu organisieren ist. In diesem Zusammenhang möchte ich die letzte Frage stellen: In welche Richtung sollte sich nach Ihrer Ansicht unsere Bewegung entwickeln; welche Fragen sollten gestellt werden, wie sollte man die Kampagne gegen den begrenzten Atomkrieg durchführen, um mehr Unterstützung zu bekommen?

Chruschtschow: Ich stimme Ihnen darin zu, dass wir jetzt einen verantwortungsvollen Punkt erreicht haben. Sie haben richtigerweise die Frage gestellt, ob nicht mehr Vertreter der Sowjetunion in der Bewegung der Anhänger des Friedens arbeiten sollten. Wir werden darüber nachdenken.

Jetzt zum „begrenzten“ Krieg. Was ist das, ein „begrenzter“ Krieg? Wer definiert ihn? Von wem hängt das ab? Die militanten Kreise der imperialistischen Mächte benutzen das Reden über den „begrenzten“ Krieg dazu, um sich die Einmischung in die Angelegenheiten kleiner Länder leicht zu machen, wie es in Guatemala geschah ${ }^{431}$ und gegen Syrien geplant ${ }^{432}$ war. Doch in jedem Krieg

427 Il'ja Ėrenburg hatte im Zarenreich der revolutionären Bewegung angehört, war unter dem Sowjetregime einer der produktivsten, bekanntesten und amtlich am meisten geschätzten Schriftsteller geworden und hatte sich im Zweiten Weltkrieg mit schärfsten antideutschen Propagandaschriften hervorgetan. 1954 war sein Buch "Tauwetter“ (Ottepel') erschienen, das der Phase größerer kultureller Freiräume nach Stalins Tod den Namen gab.

428 Aleksandr Kornejčuk war ein sowjetischer Schriftsteller aus der Ukraine, dem die Durchhalteparolen in seinen Romanen über den Zweiten Weltkrieg viermal von 1941 bis 1945 und dann nochmals 1951 den Stalin-Preis einbrachten. Er wurde damals außerdem stellvertretender Regierungschef und stellvertretender Außenminister der Sowjetrepublik Ukraine.

429 Nachdem Stalin Anfang 1949 seine Friedenskampagne eingeleitet hatte, wurde als deren Instrument auf einer international zusammengesetzten Konferenz vom 20. bis 25. April 1949 der Weltfriedensrat in Helsinki konstituiert.

430 Emmanuel d'Astier de la Vigerie spielte im Zweiten Weltkrieg eine wichtige Rolle als Verbindungsmann der französischen Résistance zu General Charles de Gaulle, der mit seinen Truppen vom westlichen Ausland aus weiter gegen die Deutschen kämpfte. Dieser ernannte ihn 1944 zum Innenminister. Mit kommunistischer Unterstützung gewann D’Astier de la Vigerie 1945 einen Wahlkreis und wurde so bis 1950 Abgeordneter in der französischen Nationalversammlung. Bei den Friedenskampagnen, die seit Anfang 1949 auf Betreiben der UdSSR durchgeführt wurden, wirkte er von Anfang an aktiv mit. 1956 übte er Kritik an der Intervention in Ungarn. Da ihn die Führung im Kreml trotzdem in der Friedensbewegung halten wollte, erhielt er im folgenden Jahr den Internationalen Lenin-Friedenspreis.

431 Aufgrund eines Wahlversprechens, das Oberst Jacobo Arbenz 1950 bei seiner Wahl zum Präsidenten von Guatemala gegeben hatte, beschloss dessen Regierung am 17. Juni 1952 eine Agrarreform, aufgrund deren nicht nur weite unbearbeitete Landflächen, sondern auch landwirtschaftliche Großbetriebe (gegen Entschädigung) den Bauern übergeben wurden. Das betraf auch die amerikanische United Fruit Company als größten Landbesitzer. Die Agrarräte, welche die Verteilung vornahmen, gerieten vielfach unter kommunistischen und anderen linken Einfluss, was in der Eisenhower-Administration, die 1953 in Washington die Regierung übernahm, zusätzliche Opposition hervorrief. Nachdem der Konflikt - auch wegen Waffenlieferungen aus der kommunistischen Tschechoslowakei - eskaliert war, intervenierte der US-Geheimdienst CIA und erzwang am 27.Juni 1954 den Amtsverzicht von Präsident Arbenz, dessen Politik anschließend revidiert wurde.

432 Seit Beseitigung der Monarchie in Ägypten 1952 bemühte sich Nasser um einen Zusammenschluss aller arabischen Länder mit dem Ziel, den amerikanischen, britischen und französischen 
gibt es zwei Seiten, von denen der Ausgang des Krieges abhängt. Die angreifende Seite kann begrenzte Ziele haben, was wir auch als lokalen Krieg bezeichnen. Solche Ziele hatten sich England und Frankreich beim Angriff gegen Ägypten ${ }^{433}$ und die USA beim Angriff gegen Guatemala gesetzt. Kürzlich haben die USA versucht, sich der ihnen unbequemen Regierung Syriens zu entledigen. Doch es kommt viel auf die Kräfte an, die gegen die Aggression Widerstand leisten. Ein Anwachsen der Kräfte, die in einen Krieg eintreten, kann zum Weltkrieg führen.

In Nord- und Südamerika gibt es keine Kräfte, die den USA Widerstand zu leisten vermögen. Die USA können bislang in dieser Region die Unterordnung der anderen Länder erreichen, ohne Gegenmaßnahmen zu befürchten. In Asien, Afrika und vor allem in Europa ist die Möglichkeit der straflosen Aggression begrenzt, wenn nicht völlig ausgeschlossen. Gegenwärtig gibt es Angaben, die Zeugnis davon ablegen, dass die konterrevolutionären Stellungnahmen in Budapest im vergangenen Jahr von einem ausländischen Geheimdienst vorbereitet worden sind. Eine große Rolle hat bei diesen Geschehnissen der englische Spionagedienst gespielt, die damit den Angriff auf Ägypten neutralisieren wollte. Die Regierungen von England und Frankreich haben darauf gehofft, der Regierung Nasser rasch ein Ende zu machen. Nur unmündige Kinder und gedankenlose Politiker können behaupten, dass die Aggression durch den UNO-Beschluss gestoppt wurde, der die Aggression verurteilt hat. Eden ist doch nicht so dumm, er ist ein gewiefter Diplomat. Schon vor dem Angriff auf Ägypten hat er gewusst, dass die UNO die Aggression verurteilen wird, doch er hat gehofft, dass man, bis die UNO zusammenkommt und diskutiert, die Regierung Nasser absetzen und eine England genehme Regierung bilden kann, um das eigene Vorgehen hinterher in der Organisation der Vereinten Nationen dadurch zu rechtfertigen, dass man auf die vorgebliche Gefahr einer Aggression seitens der Sowjetunion hinweist.

Im vergangenen Jahr ist die Aggression gestoppt worden. England und Frankreich haben sich in den Augen der Weltöffentlichkeit diskreditiert. Eden war zum Rücktritt gezwungen. Damit haben England und Frankreich infolge der Aggression in der ganzen Welt und vor allem in den arabischen und den kolonialen Ländern Prestige eingebüßt. In diesen Regionen hat man sie auch früher nicht geliebt, weil es sich um Kolonialmächte handelt, doch nach der Aggression sind sie auch in militärischer Hinsicht vom Sockel gestoßen. Die USA haben sich nach der Aggression gegen Ägypten vor den Augen der Weltöffentlichkeit auch in den arabischen Ländern politisch "betätigt". Danach ist in Syrien eine für die Vereinigten Staaten ungünstige Situation entstanden. Die USA wollten dort durch Umsturz im Innern und durch Drohungen seitens der Türkei die ihnen nicht genehme Regierung beseitigen. Doch die amerikanische Politik, das Vakuum in diesem Raum auszufüllen, ist gescheitert [und] hat ein Fiasko erlitten. Auf

Einfluss im Nahen Osten und in Nordafrika zurückzudrängen und der Existenz Israels ein Ende zu machen. Diese Ausrichtung bedrohte auch die konservativen Königreiche Saudi-Arabien, Irak und Jordanien. Unterstützung erhielt Nasser nur von Syrien, das damit zum zweiten antiwestlichen Staat neben Ägypten wurde.

${ }^{433}$ Landung britischer und französischer Truppen im Oktober 1956 zwecks Besetzung der Suezkanal-Zone, um die Verstaatlichung des Kanals durch Ägypten rückgängig zu machen. 
diese Weise ist im letzten Jahr das militärische Prestige Englands und Frankreichs [und] in diesem Jahr das militärische Prestige der USA erschüttert worden.

In Zukunft werden unsichtbare Kräfte auf jeden Fall die militärische Autorität der USA zerstören. Schließlich wissen doch alle, dass die USA der Türkei Hilfe geleistet haben, mit ihrer Kenntnis haben die Türken ihre Truppen an der syrischen Grenze konzentriert und alle Signale für den Angriff auf Syrien ausgearbeitet. Danach haben die USA der Türkei gesagt, sie sollten keine Aggression gegen Syrien beginnen. Damit haben die Vereinigten Staaten ihre militärische Autorität untergraben, sie bekamen es mit der Angst zu tun und scheuten davor zurück, die Sache bis zum Krieg gegen Syrien zu treiben, denn sie wussten, die Sowjetunion würde nicht unbeteiligt sein. Diese Lage hätte dazu führen können, dass in den Konflikt die Mitgliedsländer der NATO hineingezogen worden wären, und das hätte zum Weltkrieg führen können.

Somit ist die Politik der kleinen Kriege, der lokalen Kriege unter Anwendung der Atomwaffe darauf ausgerichtet, die öffentliche Meinung in den Ländern, die von den sozialistischen Ländern weit entfernt sind und keine Möglichkeit haben, ihre Hilfe in Anspruch zu nehmen, darauf vorzubereiten, dass diese Länder, wenn dort demokratische, den USA nicht genehme Regierungen gebildet werden, mit deren Sturz und der Einsetzung reaktionärer Regierungen zu rechnen haben, die dem Fahrwasser der Politik der USA folgen.

In Europa und Asien wird diese Politik mehr Schwierigkeiten haben, weil viele Länder an die UdSSR oder an die Länder des sozialistischen Lagers angrenzen. Ihnen kann man Hilfe leisten über die Grenze hinweg, so wie wir letztes Jahr Ungarn Hilfe geleistet haben. Wir können ebenfalls Ländern Hilfe leisten, an die wir nicht angrenzen. Als die Türkei damit begann, Syrien zu bedrohen, haben wir die Türkei gewarnt, dass wir nicht unbeteiligt bleiben werden, wenn unweit unserer Grenze ein Konflikt entsteht, und die Türken waren genötigt zurückzuweichen.

Als in Ungarn reaktionäre Kräfte die konterrevolutionäre Meuterei entfesselten, wollten England und die USA nicht hineingeraten. Einige zwei- und drittrangige Leute aus dem State Department gaben unseren diplomatischen Mitarbeitern sogar $\mathrm{zu}$ verstehen, dass sie davon überzeugt waren, die Sowjetunion werde nicht unbeteiligt bleiben, und es sei die Hilfe der Sowjetunion für Ungarn zu erwarten.

Dulles betreibt eine Politik des Manövrierens am Rande des Krieges, und solange er bei Vernunft ist, bleibt er am Rand des Krieges stehen und geht nicht darüber hinaus. Das bezeugen der türkisch-syrische Konflikt und die Ereignisse in Ungarn.

Lokale Kriege sind darauf berechnet, auf die Nerven des Gegners einzuwirken. Doch das ist eine abenteuerliche Politik. Sie erinnert mich an Akrobaten, die hoch oben unter der Zirkuskuppel arbeiten. Diese Tätigkeit endet für viele tödlich. Leute, die eine Politik der lokalen Kriege verfolgen, können ebenfalls abstürzen und Millionen von Menschen in den Abgrund eines Krieges reißen.

Was die Bewegung der Anhänger des Friedens betrifft, besonders in Ländern wie England, Frankreich, Deutschland, Dänemark, Norwegen, Belgien, Holland, Türkei, Iran, Griechenland, mit einem Wort in den Ländern, die zur NATO 
gehören,434 so müssen sie ${ }^{435}$ Verantwortung für das Schicksal ihrer Völker empfinden. Zum gegenwärtigen Zeitpunkt muss man real von den Bedingungen eines künftigen Krieges ausgehen.

Die Raketenwaffe hat das Versuchsstadium hinter sich gelassen und ist in die Arsenale der Armeen gelangt. Sie stellt für die Länder, die eine Aggression gegen uns beabsichtigen und an uns angrenzen, eine ernste Bedrohung dar. Amerikanische Politiker erklären, dass sie die Sowjetunion durch den Angriff aus vorgeschobenen Positionen vernichten werden. Sie haben dabei ihre Stützpunkte in Frankreich, England usw. im Auge. Es ist den Völkern bewusst zu machen, dass sich diese vorgeschobenen Positionen nicht in einer Wüste, sondern in Ländern mit einer dichten Besiedlung befinden. Wenn man uns von diesen Stützpunkten aus bedroht, können wir nicht gehorsam darauf warten, dass man uns vernichtet. Jeder nüchtern denkende Mensch wird verstehen, dass wir dazu genötigt sind, Gegenmaßnahmen zu ergreifen. Unsere Raketenbatterien können auf das erste Signal hin diese Stützpunkte ausschalten. Angesichts der Wasserstoffbombe besteht keine Notwendigkeit, ein genaues Ziel zu erreichen, sie vernichtet eine große Fläche. Und dann werden fürchterliche Dinge geschehen, gewaltige Räume werden verwüstet.

Die Völker dieser Länder müssen das begreifen und sich nicht selbst zum Opfer machen. Den Krieg werden die USA entfesseln und nicht England, Frankreich, Dänemark oder Norwegen. Die Vereinigten Staaten sind die Führungsmacht, überdies eine recht aggressive Führungsmacht. Zugeständnisse und Unterwürfigkeit vonseiten der Verbündeten der USA in Europa und Asien werden zu Katastrophen führen. Amerika hat auf seinem Territorium noch keinen Krieg gehabt. Doch mit den ballistischen Raketen und der Möglichkeit, sie von U-Booten aus abzuschießen, ist das Territorium der USA ebenfalls verwundbar geworden. Deswegen muss man den Menschen mit allen Mitteln dieser Gefahr bewusst machen.

Die Umstände, die es gestatten, dieser Gefahr zu entgehen, sind gut bekannt. Das ist die Politik der friedlichen Koexistenz. Wir haben wiederholt unsere Haltung in dieser Frage erklärt. Leider haben sich unsere Erklärungen noch nicht auf den Verstand mancher Politiker ausgewirkt.

Mithin sollten die Stellungnahmen der Anhänger des Friedens nicht abstrakt, sondern konkret sein.

Bernal: Mir scheint, dass gegenwärtig das Militärbündnis der NATO-Länder unter der amerikanischen Kontrolle eine große Gefahr darstellt, deswegen müssen wir uns möglichst früh dagegen wenden und eine öffentliche Meinung gegen dieses Bündnis schaffen. Für das englische Volk stellt dieses Bündnis eine unmittelbare Gefahr dar.

Chruschtschow: Ich stimme Ihnen zu. Gegenwärtig leben wir in einer gefährlichen Zeit. Mit jedem Tag werden die Waffen weiter vervollkommnet. Die Flug-

${ }^{434} \mathrm{Zu}$ diesen wird hier fälschlicherweise auch der Iran gezählt, der lediglich durch den Bagdad-Pakt mit dem NATO-Staat Türkei verbunden war.

${ }^{435} \mathrm{Im}$ russischen Protokoll steht hier der Plural statt des grammatikalisch korrekten Singulars. 
zeuge sind schon veraltet. Die ballistischen Raketen sind im Prinzip unverwundbar.

Bernal: Ihre Militärparade436 ist sehr wichtig. Die westlichen Politiker haben darauf gehofft, dass nur sie einen Atomkrieg führen können. Jetzt werden sie vorsichtiger sein.

Chruschtschow: Ich habe irgendwann einmal im Scherz gesagt, dass Amerika, England, Frankreich und die UdSSR ihre Raketen zusammen auf einem Schießplatz aufstellen und auf ein bestimmtes Ziel abfeuern sollten. Falls sich unsere Raketen als zielgenauer erweisen sollten, müssten sie einen Abrüstungsvertrag zu vernünftigen Bedingungen unterschreiben. Ich bin davon überzeugt, dass unsere Raketen nach einigen tausend Kilometern Flug genau am Ziel einschlagen würden.

Bernal: Mir wäre es lieber, wenn ein künftiger Krieg auf dem Mond stattfände.

Chruschtschow: Das wäre vielleicht das Beste. Gegenwärtig ist man genötigt, viele Mittel für Waffen zu verschwenden.

Bernal: Die kapitalistischen Länder sind außerstande, Sie mit einem direkten Angriff zu besiegen, deswegen wollen sie Sie einer Belagerung aussetzen und Sie so dazu zwingen, große Mittel für Waffen auszugeben.

Chruschtschow: Wir sind besser dran. Die Rakete kostet weniger, es werden weniger Menschen benötigt, [und] sie ist präziser, zielgenauer. Jetzt fällt das Argument Eisenhowers in Bezug auf den Überflug des Territoriums zwecks Kontrolle der Flugplätze weg. ${ }^{437}$ Für Raketen werden keine Flugplätze benötigt, ihre Startplätze sind beweglich. Jetzt bedarf es keiner Kontrolle [mehr] außer dem, was wir vorschlagen, der Kontrolle an den Orten einer möglichen Truppenkonzentration. Was die Kontrolle der Flugplätze betrifft, können die ausländischen Beobachter auf den Flugplätzen sitzen, aber die Raketen können von überall aus abgeschossen werden.

Die Öffentlichkeit muss dazu gebracht werden, gegen den Krieg einen Aufstand zu machen. Sie haben die Stimmung der Labour-Politiker richtig dargestellt. Mir hat das Gespräch mit Bevan ${ }^{438}$ gefallen. Zilliacus ${ }^{439}$ schreibt richtige, vernünftige Artikel. Manche Labour-Politiker sind freilich schlimmer als die Konservativen. Doch auch sie werden zunehmend beunruhigt sein. Das Leben

436 Jedes Jahr fanden Anfang November anlässlich des Jahrestages der russischen Oktoberrevolution auf dem Roten Platz in Moskau Militärparaden statt, bei denen man unter anderem die neuesten Waffensysteme der UdSSR präsentierte.

437 Am 21. Juli 1955 hatte Präsident Eisenhower auf der Genfer Gipfelkonferenz den Vorschlag der „offenen Himmel“ (open skies) unterbreitet. Dieser sah vor, dass die USA und die UdSSR Landkarten mit genauen Eintragungen der militärischen Einrichtungen auf ihrem Gebiet austauschten und sich gegenseitig das Recht zu Aufklärungsflügen über ihr Territorium einräumten. Die sowjetische Seite lehnte das als legalisierte Spionage ab.

438 Siehe Dokument Nr. 21 in diesem Band.

439 Konni Zilliacus war neben Aneurin Bevan der führende Politiker des linken Flügels der Labour Party. Zu seinen politischen Auffassungen siehe seine Äußerungen in den Dokumenten Nr. 16 und 27 in diesem Band. 
wird sie in die Enge treiben und die Völker werden sie dazu bringen, die Notwendigkeit der friedlichen Koexistenz anzuerkennen.

Am interessantesten ist, dass die UdSSR und die Länder des sozialistischen Lagers sich nicht nur der wirtschaftlichen, politischen und wissenschaftlichen Kontrolle durch die kapitalistischen Länder entzogen, sondern auch begonnen haben, mit ihnen in dieser Hinsicht in Wettbewerb zu treten. Das alles zwingt die Menschen im Westen dazu, über die Zukunft nachzudenken. Es gibt keinen anderen Weg - entweder Krieg oder friedliche Koexistenz. Wir werden niemals einen Krieg anfangen. Ich als Kommunist bin von den Vorzügen unserer Ordnung überzeugt und glaube, dass wir durch friedlichen Wettbewerb, durch unser Beispiel, durch die Entwicklung der Produktivkräfte den Sieg friedlich erringen werden.

Ich weiß, dass meine derartigen Erklärungen die Antikommunisten rasend machen. Doch mit der Zeit werden sie sich daran gewöhnen und sie [diese Erklärungen] vielleicht sogar zu loben anfangen.

Gen. Chruschtschow wünschte abschließend J. Bernal Erfolge bei seiner schwierigen, aber edlen Tätigkeit zum Nutzen des Friedens.

Aufgezeichnet von W. Michajlow Von Chruschtschow nicht durchgesehen

Übersetzt aus dem russischen Original RGANI, fond 52, opis‘ 1 , delo 552, Bl.65-74

\section{Chruschtschows Rede zur Eröffnung der Beratung der Vertreter der kommunistischen und Arbeiterparteien der sozialistischen Länder in Moskau am 14. November 1957440}

\section{Werte Genossen!}

Erlauben Sie mir, im Auftrag des Zentralkomitees der Kommunistischen Partei der Sowjetunion alle unsere lieben Freunde, die sich hier eingefunden haben, herzlich zu begrüßen.

Viele kommunistische und Arbeiterparteien haben es für notwendig gehalten, eine Beratung von Vertretern unserer Parteien durchzuführen, um einige der herangereiften Fragen der kommunistischen und Arbeiterbewegung zu erörtern. Unsere Parteien haben eine Übereinkunft darüber erzielt, eine solche Beratung in Moskau während der Feierlichkeiten zum 40.Jahrestag der Oktoberrevolution durchzuführen.

Und jetzt stellen wir alle mit großer Genugtuung fest, dass diese Beratung ihre Arbeit aufnimmt. Die heutige Beratung ist die bedeutendste Beratung der kommunistischen und Arbeiterparteien der sozialistischen Länder.

Es ist wohl kaum nötig zu betonen, welch eine große Bedeutung unsere Beratung hat. Wie wir schon vorab vereinbart haben, steht uns ein Meinungsaus- 